\title{
Photoactivatable Fluorescent Protein mEos2 Displays Repeated Photoactivation after a Long-Lived Dark State in the Red Photoconverted Form
}

\author{
P. Annibale, M. Scarselli, A. Kodiyan, and A. Radenovic* \\ Laboratory of Nanoscale Biology, Institute of Bioengineering, School of Engineering, EPFL, 1015 Lausanne, Switzerland
}

\begin{abstract}
Illumination with $405 \mathrm{~nm}$ light can recover the emission for single green fluorescent protein (GFP) mutants that have gone into a long-lived dark state. The reported behavior is the standard for the reverse photoswitchable protein Dronpa and its mutants. However, conventional knowledge regarding the mEos2 photoactivatable fluorescent protein (PA-FP) is that, once bleached, this fluorophore is hardly reactivated, aside from a minority population that might display this behavior. Here we show that, in a typical experiment, approximately $50 \%$ of the investigated single molecule time traces display multiple reactivations, making this a seemingly inherent feature of the mEos2 PA-FP. These results hint to some similarities between mEos 2 and other reversibly photoactivatable probes such as Dronpa. We investigate the consequences of this phenomenon in the framework of photoactivated localization microscopy (PALM) experiments.
\end{abstract}

SECTION Biophysical Chemistry

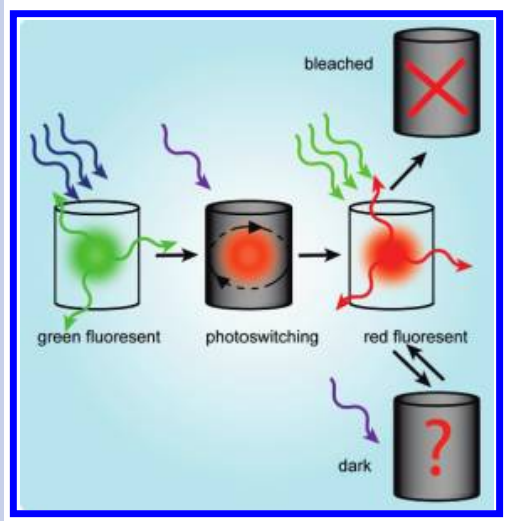

$\mathrm{D}$ evelopment in the field of super-resolution fluorescence microscopy techniques such as photoactivation localization microscopy (PALM), ${ }^{1}$ stimulated emission depletion (STED) microscopy, ${ }^{2}$ and stochastic optical reconstruction microscopy (STORM) ${ }^{3}$ spearheaded the search for brighter, more stable, and less phototoxic photoactivatable fluorescent proteins (PA-FPs) that can be selectively photoswitched from a dark to a bright state, or photoconverted from one emission wavelength to another upon stimulation with an appropriate wavelength. Among the most recently developed fluorescent proteins displaying a photoswitchable behavior, McKinney et al. $^{4}$ reported an improvement in mEosFP, a green-to-red converter, called mEos2, which has overcome the temperature sensitivity of mEos. This fluorophore displays an increased brightness and has a good photostability, targeting, and localization precision characteristics.

However, since super-resolution fluorescence microscopies are inherently single molecule fluorescence microscopy techniques, we have investigated in detail the behavior of mEos2 at the single-molecule level following the well-established approach reported by Dickson et al., ${ }^{5}$ with the goal of determining the optimal excitation/detection parameters for this molecule, and its suitability in single molecule fluorescence microscopy experiments, such as the quantitative estimation of the number of fluorophores in a diffractionlimited region, as recently reported by Greenfield et al. ${ }^{6}$ Since obtaining a controlled and tunable expression of a fluorescent protein in a biological sample is a challenging task, our experiments were performed by observing the fluorescence of purified mEos2 single molecules embedded at different concentrations in a diluted polyvinyl alcohol (PVA) aqueous solution. Although in vitro conditions are obviously different from those in a cell, a careful set of controls was put in place to rule out the possibility that our observations could be influenced by artifacts induced by the embedding polymeric matrix. Our results show that at the single molecule level a non-negligible fraction of mEos2 displays near-UVinduced reactivation, therefore hinting at the possibility that, in a single molecule counting experiment, the apparent number of mEos2 molecules could be overestimated.

To minimize this source of potential artifacts, we present an approach for determining an appropriate excitation strategy, devised first on the in vitro samples. We observed single molecular traces of the red-photo converted form of mEos2 under continuous wave (CW) $561 \mathrm{~nm}$ excitation, superposing pulses of photoactivation $405 \mathrm{~nm}$ light with different timescales, varying from 0.1 to $20 \mathrm{~s} \mathrm{CW}$ pulses. Flors et al. ${ }^{7}$ have demonstrated that the knowledge of the single molecule fluorescence behavior of the photoactivativatable fluorescent protein (PA-FP) Dronpa can be exploited toward an efficient tailoring of the excitation strategy. Since mEos2 displays a long-lived non fluorescent state, we explore how higher frequency stimulation by $405 \mathrm{~nm}$ light can affect the duration of the off-state and influence a typical PALM experiment. We present our results between the two limiting cases of broadly spaced activation pulses whose duration has been varied between $0.1 \mathrm{~s}$ and $20 \mathrm{~s}$ (see panels of Figures 1 and 5).

mEos2 is reported to undergo a transition from a greenfluorescent species to a red-fluorescent one upon $405 \mathrm{~nm}$ light

Received Date: March 17, 2010

Accepted Date: April 20, 2010

Published on Web Date: April 26, 2010 


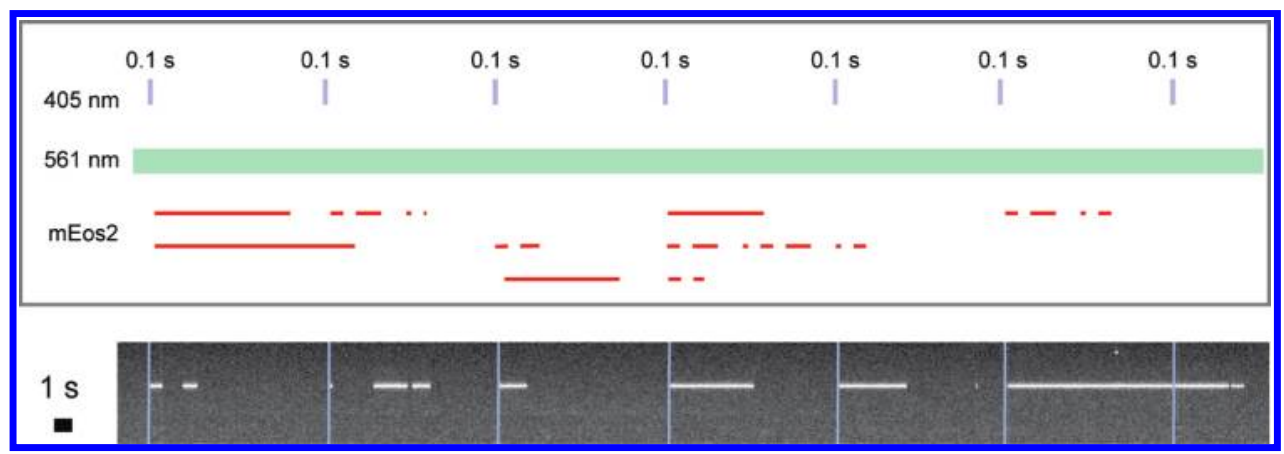

Figure 1. Kymograph of an mEos 2 molecule in a $1 \%$ weight PVA solution. The abscissa represents the time (10 pixels correspond to $1 \mathrm{~s}$ ). Vertical blue lines indicate the activation pulses at $405 \mathrm{~nm}$. The kymograph is constructed by adding up $y(t)=c$ sections taken at successive time instants. The solution is imaged under continuous $561 \mathrm{~nm}$ laser in total internal reflection fluorescence (TIRF) mode under a $60 \mathrm{X} 1.45$ NA oil immersion objective. Single-molecule fluorescence is imaged by an electron multiplying charge coupled device (EM-CCD) camera operating in frame transfer mode. Frame exposure time is set to $100 \mathrm{~ms}$.

irradiation. ${ }^{4}$ We have indeed observed the reported transition, but we also observed multiple further mEos2 reactivations from an apparently long-lived dark state. Those reactivation events are strongly correlated to the applied $405 \mathrm{~nm}$ light pulses, as can be seen in Figure 1. At the used concentrations, between $0.1 \mathrm{nM}$ and $10 \mathrm{nM}$, in a monodisperse solution, the likelihood that another molecule is found in the same pixel-sized region of another one is exceedingly low.

The largest majority of the observed traces show no detectable multistep photobleaching. This finding is indirect evidence against the presence of oligomers responsible for the observed repeated activation of a single fluorescent spot. Although, a weak dimeric character of mEos2, in particular in comparison to mEos, was reported, ${ }^{4}$ the effect is observable only at concentrations significantly higher than those employed in our study.

To further investigate and quantify how many single mEos2 molecules undergo the observed phenomenon, we proceeded by collecting statistics ${ }^{8}$ for multiple singlemolecule fluorescent traces. From the analyzed mEos 2 singlemolecule fluorescence traces we can quantify how many mEos2 molecules reactivate. The result of this analysis is plotted in the histogram displayed in Figure 2. Half of the examined molecular traces display reactivation.

As a control experiment, we also performed the same type of single-molecule photoactivation experiments on diluted samples $(0.1-10 \mathrm{nM})$ of photoactivatable green fluorescent protein (PA-GFP) ${ }^{9}$ embedded in PVA. Single-molecule kymographs for PA-GFP, under the same acquisition conditions, do not display any single-molecule reactivation behavior upon repeated $405 \mathrm{~nm}$ irradiation (see Supporting Information, Figure 2SI).

The relevance of mEos2 reactivation can be fully understood in the context of super-resolution fluorescence microscopy techniques, such as PALM-like experiments, where the potential for reactivation of fluorescent species is a significant factor to consider when planning an experiment. Although a certain degree of photochromism is commonly observed in GFP-like fluorescent proteins, the average number of times that a single PA-FP can be reactivated is obviously an important parameter to know before starting an experiment. Multiple reactivation of a fluorophore upon $405 \mathrm{~nm}$

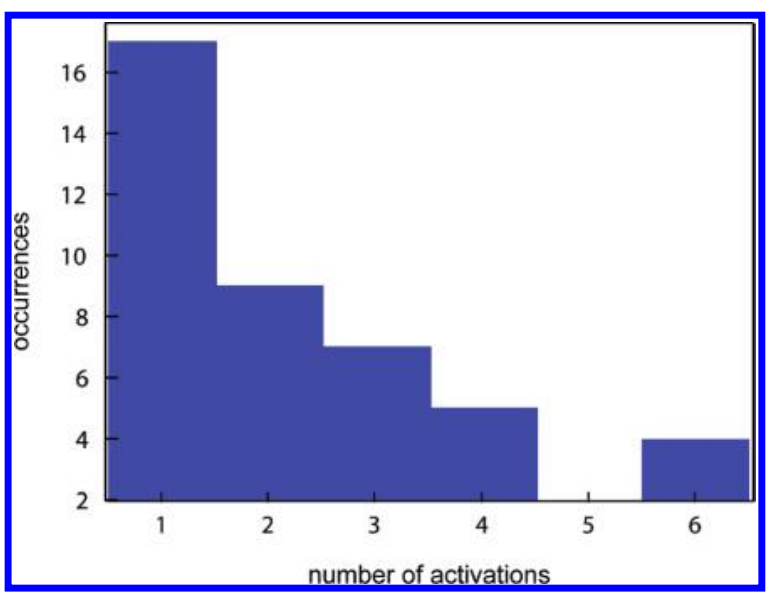

Figure 2. Histogram of the number of reactivations events per single molecule trace. Roughly half of the examined molecular traces display reactivation

excitation, e.g., Dronpa, ${ }^{10,11}$ if not taken into account, can clearly induce multiple localizations of the same fluorescent molecule, causing over counting artifacts. ${ }^{7}$ To determine whether this was indeed the case with mEos2, we performed PALM imaging obtaining a reconstructed image of the positions of the centers of all the localized molecules (Figure 3). This image was then tested for complete spatial randomness. ${ }^{12} \mathrm{~A}$ statistical test for complete spatial randomness is based on the comparison of the statistical distribution of the measured intermolecular centers distances to the theoretical distribution that would be obtained, in the same sampled area, if the centers were indeed independently distributed (see Supporting Information for further details). Since, by definition, the very low levels of activation power in PALM imaging induce a stochastic activation of only a few molecules at the time in the excitation spot, the image reconstructed at any time should satisfy the requirement for complete spatial randomness.

A bright molecule may undergo a transition to a nonfluorescent dark state, to resume its fluorescence after a certain amount of time. Well-established PALM ${ }^{1,10}$ studies take into account such time interval, that we define here as $t_{d}$, and permit to attribute a subsequent fluorescence within $t_{d}$ in the same spatial spot to the same molecule. 


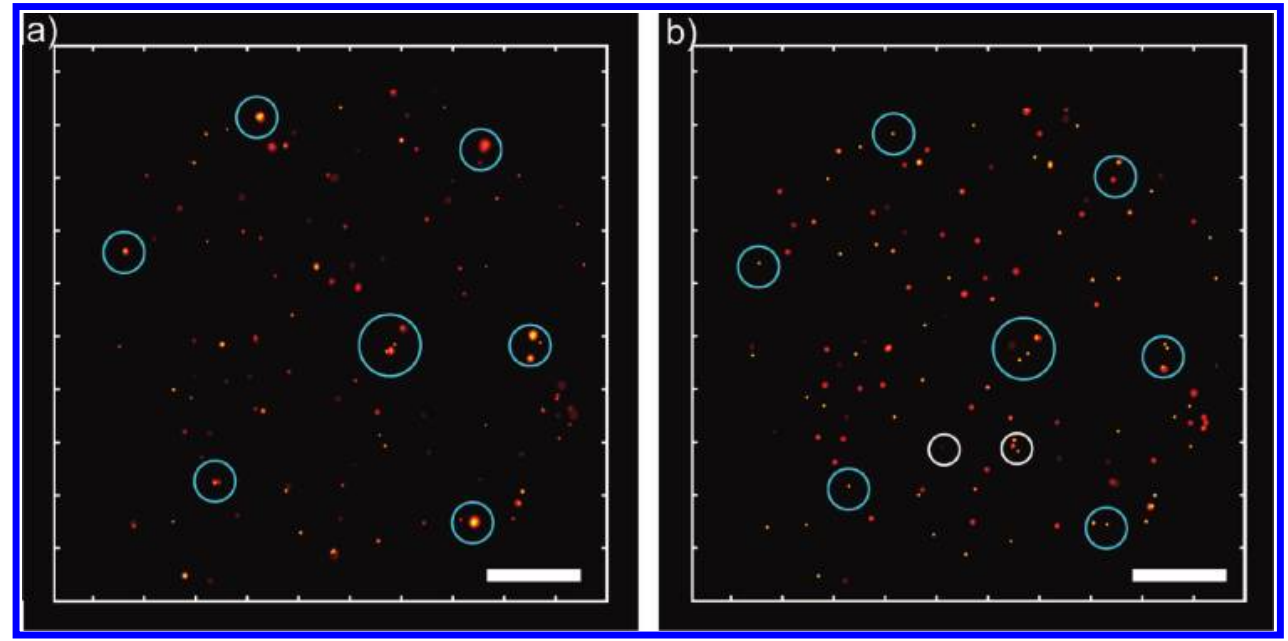

Figure 3. Comparison of two reconstructed PALM images for two different $t_{\mathrm{d}}$ (a) $5 \mathrm{~s}$ and (b) $50 \mathrm{~s}$ of mEos2 in $1 \%$ PVA solution. In the position of the center of each localized molecule, a Gaussian with height depending on the number of collected photons and $\sigma$ dependent on the signal to noise ratio was drawn. The increased intensity in panel a results from the larger number of colocalized molecules in the same spot. Blue circles indicate regions with apparent overcounting of molecules, while the white circles indicate two molecular clusters not present in panel a. Scale bar $500 \mathrm{~nm}$.

In principle, according to the originally reported ${ }^{4}$ properties of mEos2 PA-FP, we would expect that, once activated to the red-emitting state, a single molecule should quickly photobleach in a time interval corresponding to a few acquisition frames, never to be observed again. However, since our single-molecule fluorescence traces display multiple photoactivations after dark states as long as $10 \mathrm{~s}$, then it is important to investigate how the number of localized molecules in a PALM-like experiment varies with $t_{\mathrm{d}}$. As expected, a sizable decrease in the number of localized molecules is apparent. Figure 4 displays the simple exponential decay of the number of localized molecules upon the allowed time $t_{\mathrm{d}}$. A 4-fold decrease in the number of localized molecules is observed, with a $1 /$ e decay at approximately $10 \mathrm{~s}$, notably corresponding to the interpulses time interval. These results suggest that, in the presence of multiple reactivations of $\mathrm{mEos} 2$, allowing for a longer dark-state time $t_{\mathrm{d}}$ might avoid overcounting the number of molecules present in the sample.

To quantitatively estimate this phenomenon and fine-tune appropriate values for the acquisition parameters, we have performed our PALM protocol by allowing for an increasingly long $t_{\mathrm{d}}$ during which the molecule can be off, and any further emission at a later time $t<t_{\mathrm{d}}$ from the same spot will be attributed to the same molecule. We tested the resulting patterns for spatial randomness, observing a qualitative divergence from complete spatial randomness at low intermolecular distances. However, a good convergence toward complete spatial randomness can be achieved by increasing the values of the allowed $t_{\mathrm{d}}$ up to $50 \mathrm{~s}$, as demonstrated by the increasingly linear plot of the empirical distribution function $E(t)$ versus the theoretical one, as displayed in Figure 3SI (see Supporting Information). Our results point to the fact that the majority of the seemingly dimeric/oligomeric complexes or small clusters observed for low values of $t_{\mathrm{d}}$ are, in fact, artifacts due to multiple reactivations of the mEos2 molecules upon repeated $405 \mathrm{~nm}$ irradiation as outlined by the blue circles in Figure 3.

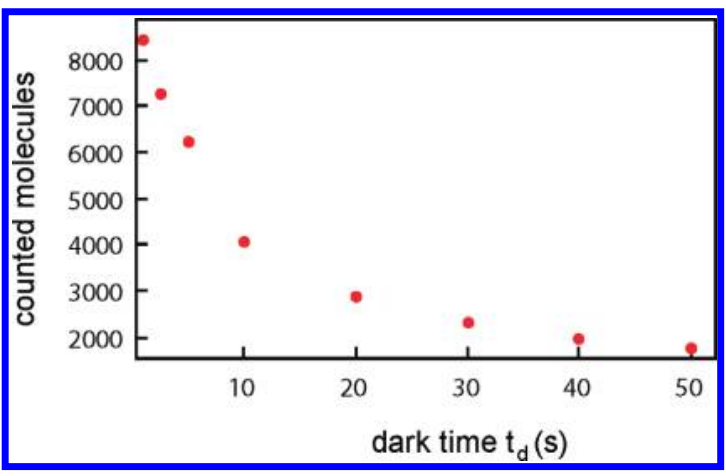

Figure 4. Amount of localized molecules as a function of the time interval $t_{\mathrm{d}}$. An inverse proportionality law clearly relates them.

The possibility of keeping molecular dark times as short as possible is an obvious advantage for any PALM experiment. We therefore performed further experiments to evaluate the effect of changing the excitation strategy on the average duration of the mEos2 long-lived dark state. From the temporally sparse short $\left(0.1 \mathrm{~s}, 1 \mathrm{~W} / \mathrm{cm}^{2}\right)$ activation pulses represented in Figure 1, we moved to longer CW activation pulses $\left(20 \mathrm{~s}, 1 \mathrm{~W} / \mathrm{cm}^{2}\right)$, separated by relatively long periods without any activation light (30 s). This approach has the advantage to decouple the effect of triplet blinking, which in principle is not reported to be affected by $405 \mathrm{~nm}$ irradiation, from an effective recovery of fluorescence from a long-lived dark state, as it happens, for example, for Dronpa. ${ }^{11}$ Tests for complete spatial randomness from the data collected under $20 \mathrm{~s} \mathrm{CW}$ pulsed activation display a qualitatively better convergence toward complete spatial randomness already at higher $t_{\mathrm{d}}$ values with respect to the data collected under pulsed activation (data not shown)

Furthermore, by direct inspection of the fluorescence traces collected under $561 \mathrm{~nm}$ excitation, with and without activation (see panel in Figure 5) it is possible to compare the effect that long $\mathrm{CW}$ activation pulses have on the on- and 


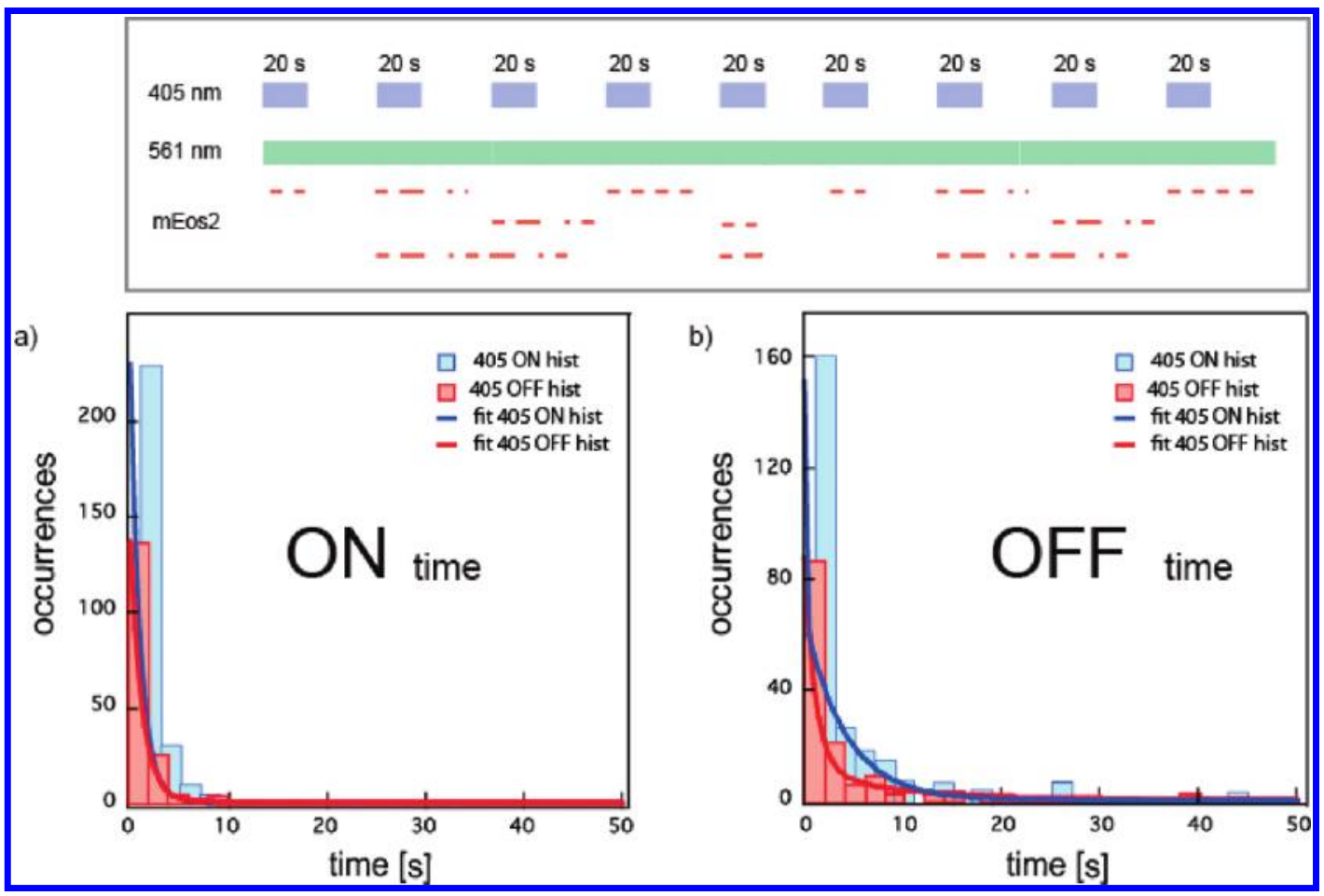

Figure 5. Top panel displays schematics of the activation strategy. (a) Histograms of the measured on-times for single molecular traces together with simple exponential fits. Red plot corresponds to traces measured when the activation light was absent, whereas blue plot corresponds to measurement intervals when a weak $20 \mathrm{~s} \mathrm{CW}$ pulsed $405 \mathrm{~nm}$ illumination was supplied. Time constants for the on-times, in the presence and absence of 405 illumination, are respectively $1.02 \pm 0.02 \mathrm{~s}$ and $1.21 \pm 0.02 \mathrm{~s}$. (b) Histograms of the measured off-times and superimposed double-exponential fits. Short time constants for the off-times, in the presence and absence of CW 405 illumination, are respectively $0.06 \pm 0.01 \mathrm{~s}$ and $1.1 \pm 0.01 \mathrm{~s}$, whereas the long off-time constants are $4.1 \pm 0.8 \mathrm{~s}$ and $8 \pm 2 \mathrm{~s}$.

off-time histograms constructed from multiple molecular traces.

Figure 5 displays the histograms of on and off times constructed by respectively analyzing 15 molecular traces from frame sequences where activation was absent and frame sequences where it was present. On-time histograms for both conditions, with and without irradiation, can be fit by simple exponentials, yielding respective time constants of $1.02 \pm 0.02 \mathrm{~s}$ and $1.21 \pm 0.02 \mathrm{~s}$. The off-time histograms, however, can be more accurately fit by a double exponential. The shorter time constant yields $0.4 \pm 0.3 \mathrm{~s}$ for the $405 \mathrm{~nm}$ irradiation condition and $1 \pm 0.2 \mathrm{~s}$ without irradiation. The longer time constant displays a shift from $4.1 \pm$ $0.1 \mathrm{~s}$ to $8 \pm 2 \mathrm{~s}$, adding evidence in favor of a $405 \mathrm{~nm}$ irradiation-driven fluorescence recovery. The effect of changing the $561 \mathrm{~nm}$ excitation power was also investigated, and the results are discussed in the Supporting Information. Preliminary data indicate that the short off-time constant appears to be affected by the $561 \mathrm{~nm}$ excitation power, whereas, within experimental error, the long off-time is not. Taken together, these results point to the fact that recovery of fluorescence of the red form of mEos 2 is elicited by $405 \mathrm{~nm}$ light, but $561 \mathrm{~nm}$ excitation light itself may play a role, as is demonstrated by the presence of an off-time constant also in the absence of activation. To draw conclusive evidence in favor of any specific photophysical model further experiments are being planned and will be the subject of a follow-up work.
In conclusion, we report here two interesting findings regarding the single-molecule behavior of the mEos2 PA-FP. The first one is that a significant fraction of mEos2 molecules can undergo multiple activations and deactivation cycles. This is an important finding since any counting experiment using this protein should take into account the possibility of multiple counts of the same molecule. The second finding is that, similarly to what was observed for Dronpa, ${ }^{11}$ constant irradiation with low-power levels (in the range of a few $\mathrm{W} / \mathrm{cm}^{2}$ ) of the activation wavelength sizably reduces the off-time duration, decreasing at the same time the chance of counting the same molecule multiple times. Although differences between the physiological conditions that can be found in either a living or fixed cell and the molecular environment present in vitro samples exist, we believe that our results show how setting the requirement for complete spatial randomness in a diluted test sample may serve as a useful control step prior to any PALM experiment in cells.

SUPPORTING INFORMATION AVAILABLE Supplementary figures and experimental methods. This material is available free of charge via the Internet at http://pubs.acs.org

\section{AUTHOR INFORMATION}

\section{Corresponding Author:}

*To whom correspondence should be addressed. E-mail aleksandra. radenovic@epfl.ch. 
ACKNOWLEDGMENT This work was supported by Swiss National Science Foundation (FNS) with Grant 200021-125319. We would also like to thank Ann McEvoy for fruitful discussion.

\section{REFERENCES}

(1) Betzig, E.; Patterson, G. H.; Sougrat, R.; Lindwasser, O. W.; Olenych, S.; Bonifacino, J. S.; Davidson, M. W.; LippincottSchwartz, J.; Hess, H. F. Imaging Intracellular Fluorescent Proteins at Nanometer Resolution. Science 2006, 313, 1642.

(2) Willig, K. I.; Kellner, R. R.; Medda, R.; Hein, B.; Jakobs, S.; Hell, S. W. Nanoscale Resolution in GFP-Based Microscopy. Nat. Methods 2006, 3, 721 .

(3) Rust, M. J.; Bates, M.; Zhuang, X. W. Sub-Diffraction-Limit Imaging by Stochastic Optical Reconstruction Microscopy (STORM). Nat. Methods 2006, 3, 793

(4) McKinney, S. A.; Murphy, C. S.; Hazelwood, K. L.; Davidson, M. W.; Looger, L. L. A Bright and Photostable Photoconvertible Fluorescent Protein. Nat. Methods 2009, 6, 131.

(5) Dickson, R. M.; Cubitt, A. B.; Tsien, R. Y.; Moerner, W. E. On/Off Blinking and Switching Behaviour of Single Molecules of Green Fluorescent Protein. Nature 1997, 388, 355.

(6) Greenfield, D.; McEvoy, A. L.; Shroff, H.; Crooks, G. E.; Wingreen, N. S.; Betzig, E.; Liphardt, J. Self-Organization of the Escherichia coli Chemotaxis Network Imaged with Superresolution Light Microscopy. PLoS Biol. 2009, 7, e1000137.

(7) Flors, C.; Hotta, J.; Uji-I, H.; Dedecker, P.; Ando, R.; Mizuno, H.; Miyawaki, A.; Hofkens, J. A Stroboscopic Approach for Fast Photoactivation-Localization Microscopy with Dronpa Mutants. L.Am. Chem. Soc. 2007, 129, 13970.

(8) Peterman, E. J. G.; Brasselet, S.; Moerner, W. E. The Fluorescence Dynamics of Single Molecules of Green Fluorescent Protein: Effect of Mutations, pH, and Matrix. Biophys. J. 1999, 76, A445.

(9) Patterson, G. H.; Lippincott-Schwartz, J. A Photoactivatable GFP for Selective Photolabeling of Proteins and Cells. $\underline{\text { Science }}$ 2002, 297, 1873.

(10) Ando, R.; Mizuno, H.; Miyawaki, A. Regulated Fast Nucleocytoplasmic Shuttling Observed by Reversible Protein Highlighting. Science 2004, 306, 1370

(11) Habuchi, S.; Ando, R.; Dedecker, P.; Verheijen, W.; Mizuno, H.; Miyawaki, A.; Hofkens, J. Reversible Single-Molecule Photoswitching in the GFP-like Fluorescent Protein Dronpa. Proc. Natl. Acad. Sci. U.S.A. 2005, 102, 9511.

(12) Statistical Analysis of Spatial Point Patterns; Diggle, P. J., Ed.; Arnold: London, 2003. 